\title{
Enhanced SLM based OFDM-DCSK communication system for PAPR reduction
}

\author{
Sajjad R. Abdulridha, Fadhil S. Hasan \\ Department of Electrical Engineering, Mustansiriyah University, Baghdad, Iraq
}

\begin{abstract}
In this research, a selective mapping (SLM) technique using the Walsh Hadamard (WH) matrix, the Riemann matrix, and the new enhanced Riemann matrix were introduced to reduce the peak to average power ratio (PAPR) of orthogonal frequency division multiplexing based differential chaos shift keying (OFDM-DCSK). Using a new algorithm to reduce the size of the Riemann matrix, which in turn enhances the computational complexity of the SLM system without affecting the performance of PAPR in the main method. The simulation results show that the SLM technique using the Riemann matrix gives better PAPR performance than WH and conventional techniques by gaining about $(6.3,6.15) \mathrm{dB}$, respectively, for complementary cumulative distribution function $(\mathrm{CCDF})=10^{-3}$ and the number of candidates $U=16$. Also, the results show that the SLM technique based enhanced Riemann matrix gives a reduction in computational complexity by reducing the number of candidates $U$ to 4 , while maintaining the same PAPR performance.
\end{abstract}

This is an open access article under the CC BY-SA license.

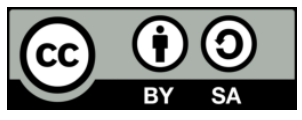

\section{Corresponding Author:}

Sajjad R. Abdulridha

Department of Electrical and Computer Engineering, Mustansiriyah University

Palastine street, Baghdad, Iraq

Email: sejjad_alqassab@yahoo.com

\section{INTRODUCTION}

Chaotic systems are widely used in wireless communication systems due to their non-periodicity, the randomness property, and the high sensibility to the initial values [1], [2]. Among all types of chaotic based modulation systems, a non-coherent chaotic modulation scheme has been highly interested by researchers due to the simplicity of the design and removing the wanted chaotic synchronization circuit at the reception. The famous practical circuit of non-coherent chaotic modulation shift keying is the differential chaos shift keying (DCSK) system [3], in which the system is designed with a simple circuit with an appropriate bit error rate (BER) under multipath fading channel without needing channel state information at the reception [4]. Nevertheless, in classical DCSK systems, only half of the bit period is consumed on carrying the data sequence [5]-[7], and the multicarrier based DCSK structure has been proposed by Kaddoum et al. [5], such as the orthogonal frequency division multiplexing (OFDM), to achieve higher information rate transmission and improve the energy efficiency of the DCSK system, which is also named multicarrier-DCSK (MC-DCSK) or OFDM-DCSK system. The subcarriers are divided into multiple groups, only one chaotic reference is sent for each group [8]. OFDM-DCSK is a non-coherent chaos-based communication structure. Improving data rate and saving bit energy are the most important advantages of this system, it's also simple to design as it does not require RF delay, and adding to the mentioned advantages, there is no need to use a chaotic sequence generator at the receiver end. The high PAPR of the transmitted signal is the major drawback of OFDM-DCSK. The RF power amplifier (RF-PA) is affected by the high PAPR, which gives rise to signal distortion. A high PAPR degrades the operation of the analog-to-digital and digital-to-analog converters and minimizes the performance 
of the RF-PA, this distorts the transmitted OFDM-DCSK signal, which leads to poor BER performance of the whole system.

The PAPR is defined as the ratio of maximum power to the mean power of the transmitted OFDMDCSK signal during one symbol. It's also expressed in units of dB. PAPR occurs in a multicarrier system when the different subcarriers are out of phase with each other. The problem of PAPR was investigated by authors and they have suggested PAPR reduction algorithms to improve the performance of the MC-DCSK systems [9]. They insert dummy sequences in accordance with the inverse fast fourier transform (IFFT) [10] needs no receiver modifications. Also, several techniques were suggested to overcome the issue of PAPR for OFDM systems, like palm date leaf clipping [11] and partial transmit sequences (PTS) [12]. A standard block code [13] was used for error correction and for OFDM PAPR reduction. In 2011, Lain et al. [14] proposed a PTS technique by using real-valued genetic algorithms to reduce the PAPR and computational complexity. PAPR reduction methods for both MRT (maximum ratio transmission) OFDM and EGT (equal gain transmission) OFDM systems were also suggested in 2014 [15], based on the obtained findings. It's worth noting that the suggested method can enhance both PAPR and the bit error rate in MRT OFDM systems, whereas it improves PAPR but only marginally decreases the bit error rate in EGT OFDM systems.

Kang et al. [16] proposed a hybrid approach of WHT precoding and companding, this approach is reducing the PAPR without affecting the performance of BER and PSD. Liu et al. [17] presented interferometry code-aided to reduce the PAPR of OFDM-DCSK. Each chaotic chip in this system is distributed over all possible subcarriers via CI codes, thereby decreasing the coherence among the signals and lowering the PAPR. The selective mapping technique (SLM) is one of the most effective techniques that has been used to reduce PAPR. Jayalath et al. [18] reduced PAPR by using Newman phase sequences. Goel et al. [19] presented the SLM technique to reduce the PAPR by using monomial phase sequences, which also reduced the computational complexity. Goel et al. [20] implemented a mixture of M-ary chaotic sequences and a mapping system. The major benefit of this technique is that it does not require side information transmission and gives good BER performance with good PAPR reduction. they [21] presented a combined PAPR reduction technique by using Riemann SLM and DCT. Mobini and Zahabi [22] presented a merit factor SLM technique to improve the PAPR reduction as compared with the conventional SLM technique. Different companding techniques were investigated, such as hyperbolic, $\mu$-Law, and $A$-Law, which were compared with the palm clipping technique to study the effects of these techniques on the PAPR of the OFDM-DCSK system and the BER performance [23]. The contribution of this paper is to reduce the PAPR value of OFDM-DCSK system by utilizing SLM technique using Hadamard, Riemann, and enhanced matrices as phase sequences and compare it with conventional SLM technique.

\section{SELECTIVE MAPPING (SLM) PAPR REDUCTION TECHNIQUES BASED OFDM-DCSK}

The PAPR is defined as the ratio of an OFDM-DCSK symbol's maximum power to the average power of that symbol.

$$
P A P R=\frac{\operatorname{Max}\left(|s(n)|^{2}\right)}{E\left\{|s(n)|^{2}\right\}}, \text { when } 0<n<N-1
$$

where $s(n)$ is the transmitted signal of the OFDM-DCSK system and $N$ is the length of the sequence. PAPR is determined by the input data. PAPR is a random variable since the input data is a random variable. The likelihood that instantaneous power is above a given threshold value can be calculated using the amplitude distribution of the OFDM-DCSK signal. The CCDF for various PAPR threshold values is used to calculate it. The efficiency of the PAPR reduction technique is conventionally used as a performance measure. The chance of an OFDM-DCSK block being more than a certain threshold value is defined as:

$$
\operatorname{CCDF}\left(P A P R_{o}\right)=\operatorname{Pr}\left(P A P R \geq P A P R_{o}\right)
$$

The threshold value and probability are defined in the above equation as $P A P R_{0}$ and $P r$ respectively.

SLM is a PAPR reduction technique that converts an OFDM-DCSK signal into many separate signals using phase sequences and transmits the signal with the lowest PAPR. For the OFDM-DCSK system, SLM is one of the distortion less PAPR reduction techniques. SLM needs the index of the chosen signal to be transmitted along with the OFDM-DCSK symbol. The sum of the change in PAPR output is determined by the number of candidates $U$ and the kind of step sequences used. The reduction in PAPR is proportional to $U$, but increasing $U$ raises side knowledge overload, which is expressed as $\left(\log _{2} U\right)$. When it comes to lowering PAPR, step sequence selection is crucial. The block diagram of the OFDM-DCSK selective mapping technique is shown in Figure 1. 


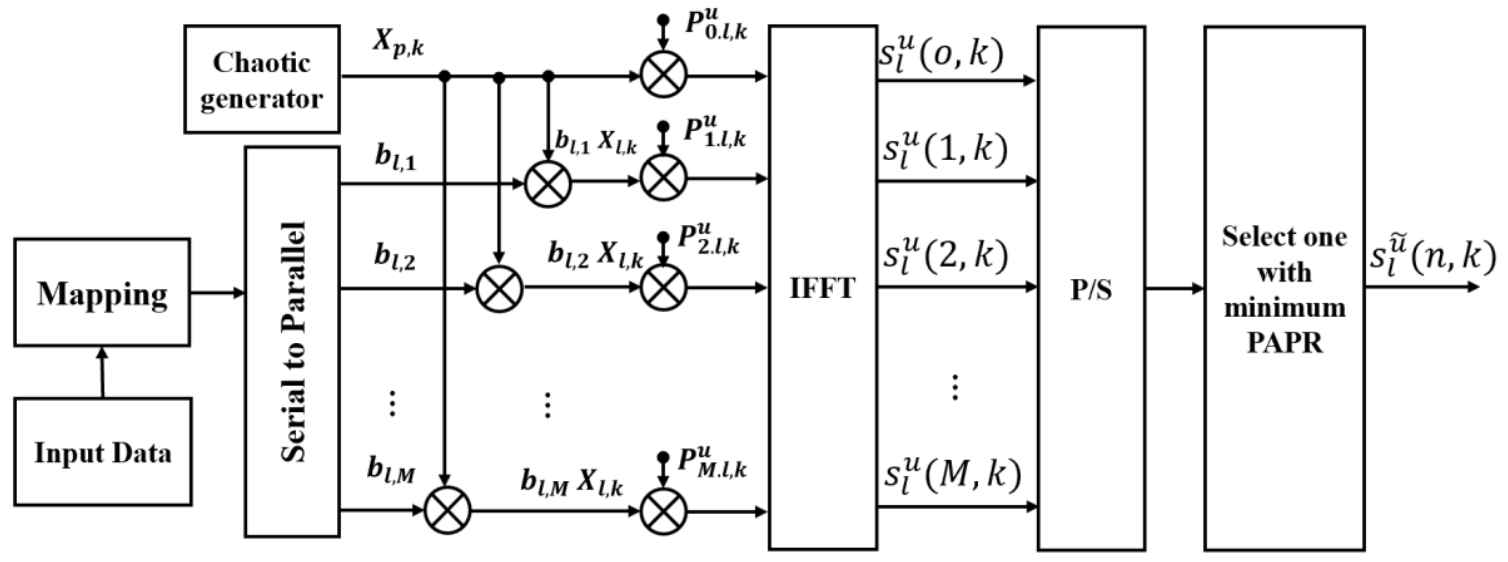

Figure 1. Block diagram of OFDM-DCSK transmitter for selective mapping technique (SLM)

In the first, the chaotic sequence is generated using second-order Chebychev Polynomial (CPF), which is given $x_{k+1}=1-2 x_{k}^{2}$ so that the $l-t h$ reference sequence is defined as $X_{l}=\left[X_{l, 0}, X_{l, 1}, \ldots \ldots \ldots \ldots, X_{l, \beta-1}\right]$, where $\beta$ is the spreading factor. The mean value for the generated sequence is zero and the mean square value is unity, $E\left[X_{l}\right]=0$ and $E\left[X_{l}^{2}\right]=1$. The $l-t h$ parallel data sequence, $b_{l, m}, m=1,2, \ldots, M \in\{-1,1\}$, is generated from the parallel mapped stream bits using a serial to parallel converter, where $M$ is the length of a parallel data sequence that are sending in each frame. The parallel data sequence is multiplied by a chaotic reference sequence and then IFFT is taken for each $k-t h$ vector $(k=0,1, \ldots, \beta-1)$ after multiplying it by the $u-t h$ phase vector, $p^{u}, u=1,2, \ldots, U$. There are $\beta$ number of IFFT transforms that are required to complete one frame. The $l-t h$ transmitted OFDM-DCSK signal and the $u-t h$ phase code can be expressed as:

$$
\begin{aligned}
& s_{l}^{u}(n, k)=\frac{1}{\sqrt{N}} \sum_{m=1}^{M} b_{l, m} X_{l, k} * e^{\left(\frac{j 2 \pi m n}{N}\right)} * p_{m . l, k}^{u}+\frac{1}{\sqrt{N}} X_{l, k} * p_{0 . l, k}^{u}, \\
& 0 \leq n<N-1, k=0,1, \ldots, \beta-1
\end{aligned}
$$

The $l-t h$ transmitted OFDM-DCSK signal which has the lowest PAPR value will be selected to be transmitted along with the $\tilde{u}$ index and the $l-t h$ transmitted signal is $\tilde{s}_{l}(n, k) . N$ is the FFT size, $N=M+1$. We assume here that the cyclic prefix is not considered. The $k-t h$ received signal is expressed as [5], [24]:

$$
r_{l}(n, k)=\sum_{i=1}^{L} \gamma_{i} \tilde{s}_{l}\left(n-\tau_{i}, k\right)+w_{l}(n, k), 0 \leq n<N-1, k=0,1, \ldots, \beta-1
$$

Where $\gamma_{i}$ and $\tau_{i}$ are the Rayleigh channel coefficient and the corresponding time delay for the $i-t h$ path, respectively. $L$ is the number of paths and $w_{l}$ is the $l-t h$ AWGN with zero mean and variance of $N_{o} / 2$. The Rayleigh pdf of $\gamma_{j}$ is written as [5]:

$$
f_{\gamma}(v)=\frac{v}{\sigma^{2}} e^{-\frac{v^{2}}{2 \sigma^{2}}}, v>0
$$

Where $\sigma$ is the standard deviation of the distribution that is greater than zero. At the receiver, non-coherent detection is used. There is no channel estimation required as in the OFDM system and no RF delay required as in the DCSK system. The $l-t h$ received signal, $r_{l}$ is converted to a parallel sequence by using a serial to parallel converter. The $l-t h$ parallel sequence, $\tilde{r}_{l, i}, i=0, \ldots, M$, are passed through the FFT transform to obtain the $m-t h$ and $k-t h$ index of the OFDM-DCSK demodulated signal at the $l-t h$ frame as:

$$
\tilde{R}_{l}(m, k)=\frac{1}{\sqrt{N}} \sum_{n=0}^{N-1} \tilde{r}_{l}(n, k) * e^{\left(\frac{-j 2 \pi m n}{N}\right) * p_{n . l, k}^{\widetilde{u}}, m=0,1, \ldots, M, k=0,1, \ldots, \beta-1}
$$

The first subcarrier (zero frequency) contains the received reference chaotic sequence, $\tilde{R}_{l}(0, k)$ and the remaining subcarrier contains the $M$ received information chaotic sequences, $\tilde{R}_{l}(m, k), m=1, \ldots, M$. There are $M$ correlators are produced from these sequences by multiplying the reference chaotic sequence by 
the $m-t h$ information sequence and summing over $\beta$ period. Then the output of the $m-t h$ correlator is given by:

$$
Q_{l, m}=\sum_{k=0}^{\beta-1} \tilde{R}_{l}(0, k) * \tilde{R}_{l}(m, k), m=1,2, \ldots, M
$$

For the $l-t h$ frame, the $m-t h$ recovered symbol is obtained by applying a decision threshold to the output correlator $Q_{l, m}$ and then the parallel symbol is converted to serial using a parallel to serial converter. Finally, the recovered stream bits are obtained using the Demapping function by mapping +1 to 1 and -1 to 0 .

\section{PHASE CODE GENERATION}

When it comes to lowering PAPR, phase sequence selection is crucial. In this section, the phase code sequences are discussed.

\subsection{Random phase code}

Multiple modified data sequences are generated by multiplying random sequences with the original signal. The random sequences are generated by using phase sequences, which contain different rotation factors. The original signal phase was rotated by using these factors to reduce the PAPR. The $U$ new generated sequences, which is statistically independent are forwarded into IFFT operations, then the PAPR will be calculated for each one to select the lowest value for transmission. The $u-t h$ generated statistically independent phase sequences are expressed as:

$$
P^{(u)}=\left[p_{0}^{u}, p_{1}^{u}, p_{2}^{u}, \ldots, p_{N-1}^{u}\right], u=1,2, \ldots, U
$$

The $u-t h$ generated phase sequence is multiplied by the OFDM-DCSK symbol. The $p_{m}^{u}$ represents the $m-$ th phase in the $u-t h$ code, which is given by:

$$
p_{m}^{u}=e^{j \phi_{m}^{u}} \text {, where } \emptyset_{m}^{u} \in[0,2 \pi), m=0,1, \ldots, N-1 \text { and } u=1,2, \ldots, U
$$

The different random phase sequences are multiplied with the data symbol. The obtained sequences are denoted as candidates. The time domain signal is generated after taking IFFT for each candidate. After that, the PAPR of all time domain candidates is calculated. The lower PAPR is selected to be transmitted with the phase sequence which is necessary to recover the signal at the receiver. This side information is considered as the main drawback of this technique.

\subsection{Hadamard phase code}

In this technique, instead of random multiplying sequences, Hadamard codes were selected, which are perfectly orthogonal. This will improve the probability of achieving a better PAPR reduction with a small number of sequences. The Hadamard code is a square matrix of rows that are mutually orthogonal and entries which are either +1 or -1 . This implies that each pair of rows in a Hadamard matrix represents two perpendicular vectors in geometric terms, and each pair of rows has identical entries in precisely half of their columns and mismatched entries in the remaining columns in combinatorial terms. As a result of this description, the related properties apply to all columns and rows.

$$
H_{1}=[1], H_{2}=\left[\begin{array}{cc}
1 & 1 \\
1 & -1
\end{array}\right]
$$

So, the Hadamard matrix is denoted as:

$$
\begin{aligned}
& H_{2^{k}}=\left[\begin{array}{cc}
H_{2^{k-1}} & H_{2^{k-1}} \\
H_{2^{k-1}} & -H_{2^{k-1}}
\end{array}\right] \\
& H=\left[\begin{array}{ccc}
h_{11} & \cdots & h_{1 N} \\
\vdots & \ddots & \vdots \\
h_{N 1} & \cdots & h_{N N}
\end{array}\right]
\end{aligned}
$$

The $m-t h$ phase in the $u^{\text {th }}$ code depending on Hadamard matrix is given by: 


$$
p_{m}^{u}=h_{m, u}, m=1,2, \ldots, N, u=1,2, \ldots, N
$$

Different $U$ orthogonal independent vectors with a length equal to $N$ were selected from the Hadamard matrix to multiply them with the data signal to generate the candidate sequences. The time domain signal is generated after taking IFFT for each candidate. After that, the PAPR of all time domain candidates is calculated. The candidate with the lowest PAPR was selected to be transmitted. The value of the index (column index) is needed to be transmitted as side information to recover the original data block by doing the reverse process at the receiver.

\subsection{Riemann phase code}

In this technique, the SLM technique is achieved by using the column phases of Riemann [25], which are considered as a phase sequences. The Riemann matrix is obtained by neglecting the first row and first column of the $M \times M$ matrix, where:

$$
R(i, j)=\left\{\begin{array}{cc}
i-1 & \text { if } i \text { divides } j \\
-1 & \text { otherwise }
\end{array}\right.
$$

The element value of the $u-t h$ column is either -1 , which causes a phase change or $1 \leq u \leq N$, which causes a change in the amplitude value of the modulated symbol.

\subsection{Enhanced Riemann matrix}

In this suggested technique, a MATLAB program is used to reduce the size of Riemann matrix and find the optimal $U$ vectors that result in a lower PAPR and to generate a new matrix containing $U$ columns that result in lower computational complexity and optimal PAPR reduction. Below is the algorithm for the suggested technique, and also the algorithm is shown in figure 2.

a. Multiply the OFDM-DCSK signal with all Riemann column vectors.

b. Taking the IFFT for all the generated sequences.

c. Calculate the PAPR for all time domain sequences.

d. Select the first $U$ Riemann phase codes that have the minimum PAPR values.

e. Generate the new enhanced matrix of $U$ columns.

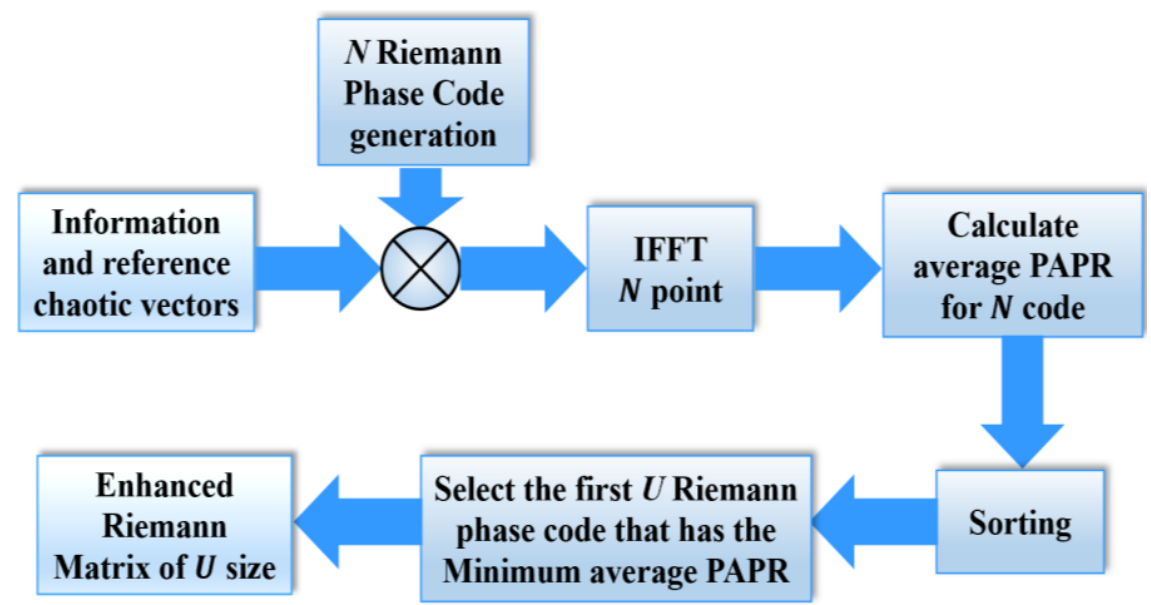

Figure 2. Enhanced Riemann phase code based SLM technique

\section{SIMULATION RESULTS AND DISCUSSIONS}

Simulation results were taken in this section to evaluate the performance of the SLM technique by using Walsh Hadamard, Riemann, and the Enhanced Riemann matrix and comparing it with the conventional SLM technique based on computer simulations. In this work, we built the MATLAB model of the system over two path Rayleigh fading channel, $L=2$, are used with delays, $\tau_{1}=0$ and $\tau_{2}=2 T_{c}$ and average power gain, $E\left[a_{1}^{2}\right]=\frac{2}{3}$ and $E\left[a_{2}^{2}\right]=\frac{1}{3}$, the number of subcarriers $N$ is set to 256 and 512 , the spreading factor $\beta$ is set to 64 , and the number of sequences $U$ is selected to be 4,8 , and 16 . The PAPR reduction performance comparison of the proposed techniques was studied, while setting the SNR value to $10 \mathrm{~dB}$. Figure 3 and Figure 4 describe 
the gain of the tried techniques in terms of PAPR at CCDF $=10^{-2} \& 10^{-3}$ for $N$ is equal to 256 and 512 respectively. It was noticed that the proposed technique results in a PAPR gain of approximately $9.6 \mathrm{~dB}$ and $10 \mathrm{~dB}$ at $\mathrm{CCDF}$ equal to $10^{-3}$ when the number of subcarriers is equal to 256 and 512 respectively, which gives better results than other techniques for both PAPR gain value and number of candidates $(U)$. It was noticed that WH SLM and conventional SLM approximately have the same PAPR value reduction, but in the WH technique there is no need to send side information, which is necessary in the conventional SLM technique to recover the original signal at the receiver. Sending the $\tilde{u}$ index is enough to recover the original signal at the receiver for WH, Riemann, and the Enhanced Riemann techniques. Finally, the results of the performance evaluation are listed for all the techniques that were used in our work in Tables 1 and 2 for $N=256$ and 512 , respectively. The included results are obtained for a different number of $N$ by setting the spreading factor $(\beta)$ to 64. The Tables 1 and 2 present the comparison between the techniques in terms of $P A P R_{E f}$.

$$
P A P R_{E f}=P A P R_{\text {original }}-P A P R_{\text {new }}
$$

Table 1. Comparative study of the suggested PAPR reduction techniques for $P A P R_{E f}$ at $N=256, \beta=64$

\begin{tabular}{ccccccc}
\hline & $P A P R_{E f}(\mathrm{~dB})$ at $\mathrm{CCDF}=10^{-2}$ & \multicolumn{4}{c}{$P A P R_{E f}(\mathrm{~dB})$ at $\mathrm{CCDF}=10^{-3}$} \\
& $U=4$ & $U=8$ & $U=16$ & $U=4$ & $U=8$ & $U=16$ \\
\hline Conventional SLM & 1.75 & 2.3 & 2.7 & 2.35 & 2.975 & 3.45 \\
WH SLM & 1.75 & 2.275 & 2.7 & 2 & 2.8 & 3.3 \\
Riemann SLM & 8.5 & 8.66 & 8.8 & 9.25 & 9.47 & 9.6 \\
Enhanced Riemann SLM & 8.8 & -- & -- & 9.6 & -- & -- \\
\hline
\end{tabular}

Table 2. Comparative study of the suggested PAPR reduction techniques for $P A P R_{E f}$ at $N=512, \beta=64$

\begin{tabular}{ccccccc}
\hline & \multicolumn{3}{c}{$P A P R_{E f}(\mathrm{~dB})$ at $\mathrm{CCDF}=10^{-2}$} & \multicolumn{4}{c}{$P A P R_{E f}(\mathrm{~dB})$ at $\mathrm{CCDF}=10^{-3}$} \\
& $U=4$ & $U=8$ & $U=16$ & $U=4$ & $U=8$ & $U=16$ \\
\hline Conventional SLM & 1.5 & 2 & 2.35 & 2 & 2.6 & 3.05 \\
WH SLM & 2 & 2.4 & 2.775 & 2.4 & 2.95 & 3.4 \\
Riemann SLM & 9.05 & 9.15 & 9.2 & 9.8 & 9.95 & 10 \\
Enhanced Riemann SLM & 9.2 & -- & -- & 10 & -- & -- \\
\hline
\end{tabular}

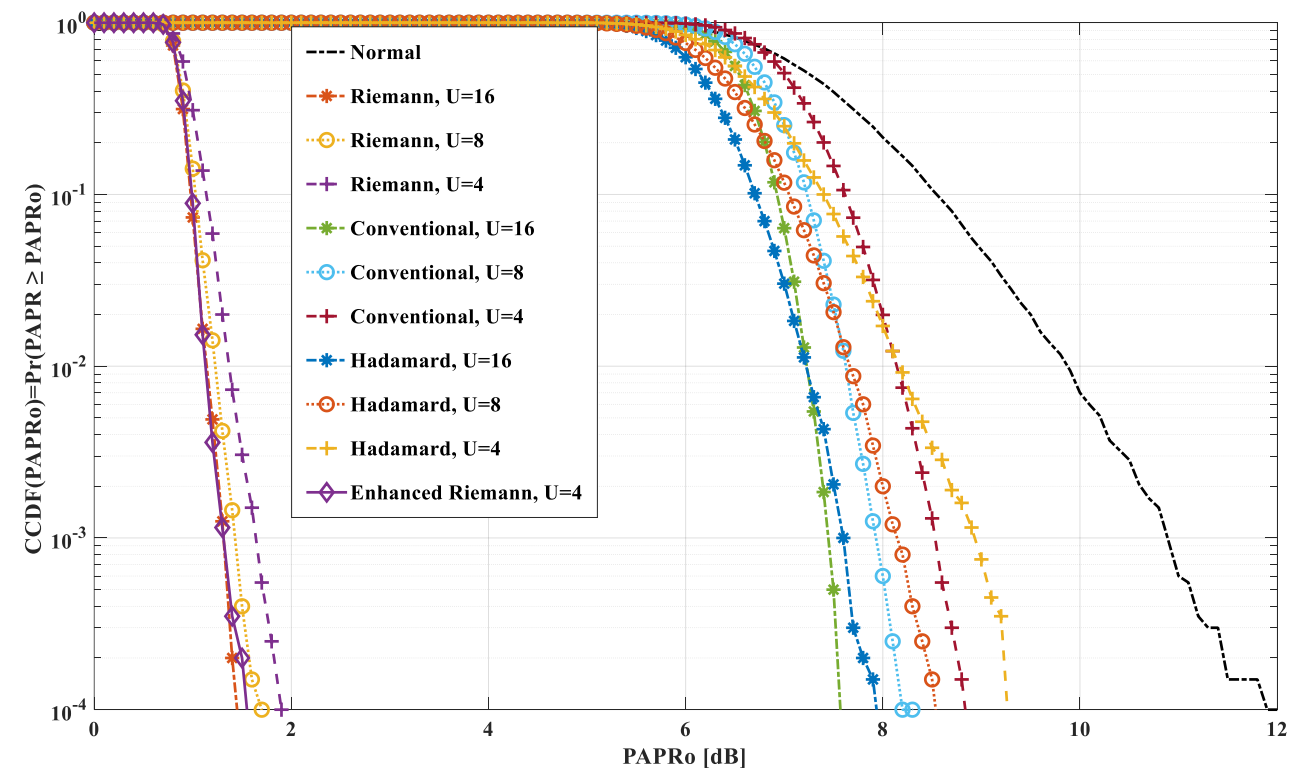

Figure 3. PAPR comparisons performance for all the tried techniques for different number of candidates, $N=256$ and $\beta=64$ 




Figure 4. PAPR comparisons performance for all the tried techniques for different number of candidates, $N=512$ and $\beta=64$

\section{CONCLUSION}

In this paper, SLM techniques using WH, Riemann, and the Enhanced Riemann matrix were performed to overcome the issue of high PAPR, which affects the efficiency of the power amplifier. The PAPR reduction was done by multiplying the OFDM-DCSK signal with different sequences and selecting the one which gives a lower PAPR value before transmitting the signal over the multipath Rayleigh fading channel. The inverse process was used at the receiver to recover the original signal by using the $\tilde{u}$ index. The simulation results obtained by running the MATLAB program show that the PAPR reduction value has improved as a result of $P A P R_{E f}$. The SLM based enhanced Riemann matrix gives the best results compared to the other tried techniques in terms of $P A P R_{E f}$ value with a smaller number of candidates $U$, which in turn decreases the processing time from 3.536 to 0.884 seconds. The elapsed time for each candidate is 0.221 seconds, which was calculated by MATLAB program.

\section{REFERENCES}

[1] F. C. M. Lau and C. K. Tse, "Chaos-Based Digital Communication Systems”. New York, NY: Springer, 2003.

[2] H. Dedieu, M. P. Kennedy and M. Hasler, "Chaos shift keying: modulation and demodulation of a chaotic carrier using selfsynchronizing Chua's circuits," in IEEE Transactions on Circuits and Systems II: Analog and Digital Signal Processing, vol. 40, no. 10, pp. 634-642, Oct. 1993, doi: 10.1109/82.246164.

[3] G. Kolumban, B. Vizvki, W. Schwarz, and A. Abel, "Differential chaos shift keying: A robust coding for chaotic communication," Proceeding 4th International Work. Nonlinear Dynamic Electron System, pp. 87-92, 1996.

[4] Y. Fang, G. Han, P. Chen, F. C. M. Lau, G. Chen and L. Wang, "A Survey on DCSK-Based Communication Systems and Their Application to UWB Scenarios," in IEEE Communications Surveys \& Tutorials, vol. 18, no. 3, pp. 1804-1837, thirdquarter 2016, doi: 10.1109/COMST.2016.2547458.

[5] G. Kaddoum, F. Richardson and F. Gagnon, "Design and Analysis of a Multi-Carrier Differential Chaos Shift Keying Communication System," in IEEE Transactions on Communications, vol. 61, no. 8, pp. 3281-3291, August 2013, doi: 10.1109/TCOMM.2013.071013.130225.

[6] T. Huang, L. Wang, W. Xu and G. Chen, "A Multi-Carrier $M$-Ary Differential Chaos Shift Keying System With Low PAPR," in IEEE Access, vol. 5, pp. 18793-18803, 2017, doi: 10.1109/ACCESS.2017.2752238.

[7] S. Li, Y. Zhao, and Z. Wu, "Design and analysis of an OFDM-based differential chaos shift keying communication system," Journal of Communication, vol. 10, no. 3, pp. 199-205, 2015, doi: 10.12720/jcm.10.3.199-205.

[8] G. Kaddoum, "Design and Performance Analysis of a Multiuser OFDM Based Differential Chaos Shift Keying Communication System," in IEEE Transactions on Communications, vol. 64, no. 1, pp. 249-260, Jan. 2016, doi: 10.1109/TCOMM.2015.2502259.

[9] G. Cai, Y. Fang, G. Han, F. C. M. Lau and L. Wang, "A Square-Constellation-Based M -Ary DCSK Communication System," in IEEE Access, vol. 4, pp. 6295-6303, 2016, doi: 10.1109/ACCESS.2016.2612224.

[10] Heung-Gyoon Ryu, Jae-Eun Lee and Jin-Soo Park, "Dummy sequence insertion (DSI) for PAPR reduction in the OFDM communication system," in IEEE Transactions on Consumer Electronics, vol. 50, no. 1, pp. 89-94, Feb. 2004, doi: 10.1109/TCE.2004.1277845.

[11] B. Bakkas, R. Benkhouya, I. Chana, and H. Ben-Azza, "Palm date leaf clipping: A new method to reduce PAPR in OFDM systems," Information, vol. 11, no. 4, 190, 2020, doi: 10.3390/info11040190. 
[12] Y. A. Jawhar et al., "A Review of Partial Transmit Sequence for PAPR Reduction in the OFDM Systems," in IEEE Access, vol. 7, pp. 18021-18041, 2019, doi: 10.1109/ACCESS.2019.2894527.

[13] I. Shakeel and A. Grant, "Joint Error Correction and PAPR Reduction of OFDM Signals," 2006 IEEE Information Theory Workshop - ITW'06 Chengdu, 2006, pp. 611-615, doi: 10.1109/ITW2.2006.323707.

[14] J. K. Lain, S. Y. Wu, and P. H. Yang, "PAPR reduction of OFDM signals using PTS: a real-valued genetic approach," EURASIP Journal on Wireless Communications and Networking, vol. 2011, no 126, pp. 1-8, 2011, doi: 10.1186/1687-1499-2011-126.

[15] Y. -C. Hung and S. -H. L. Tsai, "PAPR Analysis and Mitigation Algorithms for Beamforming MIMO OFDM Systems," in IEEE Transactions on Wireless Communications, vol. 13, no. 5, pp. 2588-2600, May 2014, doi: 10.1109/TWC.2014.031914.130347.

[16] C. Kang, Y. Liu, M. Hu and H. Zhang, "A Low Complexity PAPR Reduction Method Based on FWFT and PEC for OFDM Systems," in IEEE Transactions on Broadcasting, vol. 63, no. 2, pp. 416-425, June 2017, doi: 10.1109/TBC.2016.2637278

[17] Z. Liu, L. Zhang and Z. Chen, "Low PAPR OFDM-Based DCSK Design With Carrier Interferometry Spreading Codes," in IEEE Communications Letters, vol. 22, no. 8, pp. 1588-1591, Aug. 2018, doi: 10.1109/LCOMM.2018.2842196.

[18] A. D. S. Jayalath, C. Tellambura and H. Wu, "Reduced complexity PTS and new phase sequences for SLM to reduce PAP of an OFDM signal," VTC2000-Spring. 2000 IEEE 51 st Vehicular Technology Conference Proceedings (Cat. No.00CH37026), 2000, pp. 1914-1917 vol.3, doi: 10.1109/VETECS.2000.851605.

[19] G. T. Zhou, R. J. Baxley and N. Chen, "Selected mapping with monomial phase rotations for peak-to-average power ratio reduction in OFDM," 2004 International Conference on Communications, Circuits and Systems (IEEE Cat. No.04EX914), 2004, pp. 66-70 Vol.1, doi: 10.1109/ICCCAS.2004.1345941.

[20] A. Goel, M. Agrawal, and P. G. Poddar, "M-ary Chaotic Sequence Based SLM-OFDM System for PAPR Reduction without SideInformation,” World Acad. Sci. Eng. Technol. Int. J. Inf. Commun. Eng., vol. 6, no. 8, pp. 783-788, 2012.

[21] P. Magnangana, Z. Goyoro, I. J. Moumouni, and S. Abouty, "SLM Using Riemann Sequence Combined with DCT Transform for PAPR Reduction in OFDM Communication Systems," International Journal of Information and Communication Engineering, vol. 6, no. 4, pp. 394-399, 2012.

[22] M. Mobini and M. R. Zahabi, "A Low Complexity PAPR Reduction Architecture for OFDM-DSCK Communication System," Wireless Personal Communications, vol. 107, no. 4, pp. 1759-1776, 2019, doi: 10.1007/s11277-019-06355-5.

[23] S. R. Abdulridha and F. S. Hasan, "Palm Clipping and Nonlinear Companding Techniques Based Papr Reduction in Ofdm-Dcsk System," Journal of Engineering and Sustainable Development, vol. 25, no. 4, pp. 84-94, 2021, doi: 10.31272/jeasd.25.4.8.

[24] F. S. Hasan and A. A. Valenzuela, "Design and analysis of an OFDM-Based orthogonal chaotic vector shift keying communication system," IEEE Access, vol. 6, pp. 46322-46333, 2018, doi: 10.1109/ACCESS.2018.28628.

[25] Friedrich Roesler, "Riemann's hypothesis as an eigenvalue problem," Linear Algebra and its Applications, vol. 81, pp. 153-198, 1986, doi: 10.1016/0024-3795(86)90255-7.

\section{BIOGRAPHIES OF AUTHORS}



Sajjad R. Abdulridha (D) SC P Pas born in Baghdad, Iraq in 1987. He received the engineer degree in electrical engineering in 2009 from the Mustansiriyah University, Iraq. His recent research is to reduce the peak to average power ratio (PAPR) of orthogonal frequency division multiplexing based different caotic shift keying (OFDM-DCSK) system using distortion and distortion-less techniques. He can be contacted at email: sejjad.alqassab@uomustansiriyah.edu.iq.

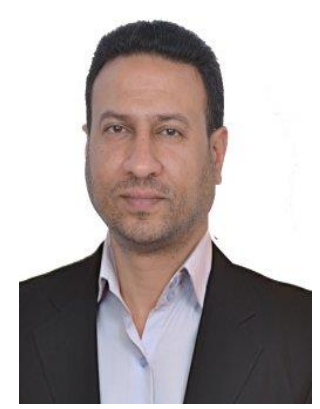

Fadhil S. Hasan (D) 8d SC P was born in Baghdad, Iraq in 1978. He received his B.Sc. degree in electrical engineering in 2000 and his M.Sc. degree in electronics and communication engineering in 2003, both from the Mustansiriyah University, Iraq. He received Ph.D. degree in 2013 in electronics and communication engineering from the Basrah University, Iraq. In 2005, he joined the faculty of Engineering at the Mustansiriyah University in Baghdad. His recent research activities are Wireless communication systems, multicarrier system, wavelet based OFDM, MIMO system, speech signal processing, image signal processing, cryptography and computer security, chaotic modulation, FPGA and Xilinx system generator-based communication system. Now he has been an Assist. Prof. at the Mustansiriyah University, Iraq. $\mathrm{He}$ can be contacted at email: fadel`sahib@uomustansiriyah.edu.iq. 